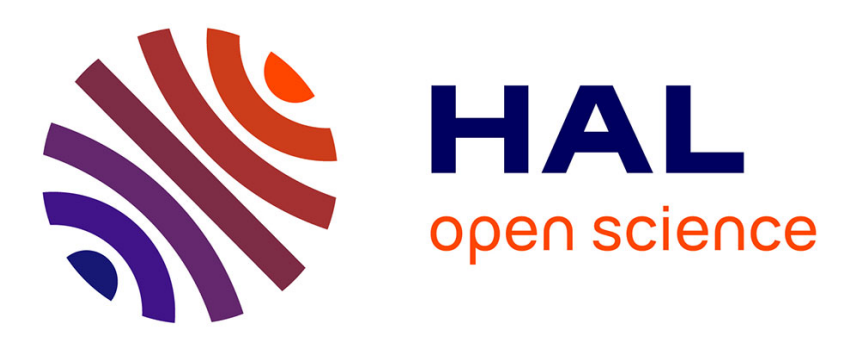

\title{
Weakly intrusive time homogenization technique to deal with pseudo-cyclic coupled thermomechanical problems with uncertainties
}

Chenchen Chu, Mainak Bhattacharyya, David Dureisseix, Béatrice Faverjon

\section{- To cite this version:}

Chenchen Chu, Mainak Bhattacharyya, David Dureisseix, Béatrice Faverjon. Weakly intrusive time homogenization technique to deal with pseudo-cyclic coupled thermomechanical problems with uncertainties. Computational Mechanics, 2020, 66 (3), pp.669-682. 10.1007/s00466-020-01871-9 . hal02889966v2

\section{HAL Id: hal-02889966 \\ https://hal.science/hal-02889966v2}

Submitted on 14 May 2021

HAL is a multi-disciplinary open access archive for the deposit and dissemination of scientific research documents, whether they are published or not. The documents may come from teaching and research institutions in France or abroad, or from public or private research centers.
L'archive ouverte pluridisciplinaire HAL, est destinée au dépôt et à la diffusion de documents scientifiques de niveau recherche, publiés ou non, émanant des établissements d'enseignement et de recherche français ou étrangers, des laboratoires publics ou privés. 


\title{
Weakly intrusive time homogenization technique to deal with pseudo-cyclic coupled thermomechanical problems with uncertainties
}

\author{
Chenchen $\mathrm{Chu}^{1}$, Mainak Bhattacharyya ${ }^{2}$, David Dureisseix ${ }^{1}$, and Béatrice Faverjon ${ }^{1}$ \\ ${ }^{1}$ Univ Lyon, INSA-Lyon, CNRS UMR5259, LaMCoS, F-69621, France \\ ${ }^{2}$ LMT, ENS Paris-Saclay, CNRS, Université Paris Saclay, F-94235 Cachan Cedex, \\ France
}

\begin{abstract}
This article is dedicated to the analysis of visco-plastic and damageable structures submitted to thermomechanical loadings embedding some uncertain parameters. In particular, complex time evolutions of the loadings are considered, at two different time scales. An homogenized-in-time model is used to provide kind of a surrogate model for damage evolution analysis. Then a stochastic analysis using polynomial chaos expansion with collocation can be performed. Indeed, though this is a non-intrusive approach, it belongs to the category of many-query problems, so the surrogate model allows to reduce the computational cost in order to be affordable. Focus is herein on the complex time evolutions, the considered test case is mono-dimensional, but up to 300000 cycles are used.

This is a post-peer-review, pre-copyedit version of an article published in Computational Mechan$i c s$. The final authenticated version is available online at:

http://dx.doi.org/10.1007/s00466-020-01871-9

Note that the figure numbering in the editor final version is not correct.
\end{abstract}

Keywords: Parametric uncertainty quantification, Time multiscale, Damage, Fatigue

\section{Introduction}

Engines are typically made with some structural assembling that encounters a complex loading history. The mechanical (and possibly multiphysical) loads are often composed of high frequency cyclic evolutions, together with low frequency modulations in terms of mean or amplitude [4, 3]. The case under concern is herein the cumulative damage and visco-plasticity due to quasi-static thermomechanical loadings.

These phenomena can also occur for different families of problems that concern materials and structures for which there exist a cumulative effect at short scale in time, that may have an influence on the structure behavior. Such a class of problems contains for instance:

- fatigue at high number of cycles [32, 15, 20],

- differential settlement of railway ballast [46, 45],

- wear influence on geometry [27, 38],

- bone remodeling [13, 39], etc.

There are several available approaches to deal with different time scales in structural behavior, such as $[23,7,53,29]$. Among numerical strategies, one can find cycle jump techniques [9, 11, 6, 10, $35,28]$, or semi or full decoupling between time scales [33, 31].

In such cases, the behavior of overall structure may be described with two time scale evolutions. Indeed, for linear behavior, a superposition principle can be used to access the full solution. For nonlinear cases, the situation is more challenging, and we aim to use an homogenization-like procedure (first used in [24]) to derive a macroscopic-in-time behavior that can be corrected by a microscopicin-time evolution at the underlying scale $[43,41,20]$. We take advantage of the quasi-periodic assumption at high-frequency (denoted herein as microscopic time evolution) to decouple the micro evolution from the overall time evolution. Indeed; dealing with a time step for the discretization driven by the high frequency loading on all the studied time evolution would lead (as the reference 
direct problem) to a very large number of time steps, inversely proportional to the time scale ratio. Without scale separation, this is the topic of coupling different discretizations in time [40, 12, 17].

When the time scale ratio is small, the reference problem is very costly, but the two time scales are more and more decoupled, so the quasi-periodic assumption is more and more valid. This method has been used for classical elasto-viscoplasticity in [43, 44], viscoelasticity in [52]. As far as damage is concerned, it has been used for elasticity-based damage cases in [13], for Gurson-TvergaardNeedleman based damage models in [42, 21] and for a viscoplastility-like damage model in [41]. Recently we have used this method in combination with cycle jump approach for the simulation of combined cycle fatigue [2]. The new issues herein, apart from application to stochastic analysis, is the coupling of damage with multiphysics (thermo-mechanical behavior, with two different natures of problems) and viscoplasticity with a general formulation including both kinematic and isotropic hardening. We nevertheless restrict this analysis to quasi-static regime, and not with linear wave propagation such as in [54]. The non-linear material is herein damageable, but its evolution is progressive in time.

Periodicity assumption allows to decouple the micro cycles one to the other. This is an interesting feature in spatial homogenization, where elliptic spatial micro problems are decoupled. In such cases, two scales in space also allow to compute the micro solutions only under macroscopic Gauss points (the FE2 technique $[19,18]$ ) so that not all micro cells have to be considered (higher order micro boundary condition is herein the equivalent of macro values and macro gradient values).

Simply decoupling the micro cycles in time from one to another allows parallelization [37, 14] but leads to no gain (in cumulative CPU time), since the reference problem is parabolic in a onedimensional time domain. Therefore, two-time-scale models also provide a cycle jump technique since the micro-cycles are computed only when required at macroscopic time steps. Large macroscopic time steps therefore involves a saving in the number of effectively computed micro cycles.

In this study, we focus on weakly coupled thermo-mechanical problems with coupled viscoplasticity and damage. Concern is also dove on non-intrusive approaches, in order to be able to reuse as far as possible pre-existing codes, contrary to proper generalized decomposition (PGD) techniques $[4,47]$. As modeling is performed at small scales, the effects are usually sensitive to defects in the material, structure, or boundary conditions, or more generally to uncertainties in the model. To cope with this feature, we also propose to embed a non-intrusive stochastic modeling in conjunction with the two time scale strategy. This modeling is based on a probabilistic collocation method described in $[48,26,25]$. The non intrusive aspect of the approach has the advantage of using a black box independently of the stochastic modeling, and its efficiency for coupled problems has been showed in [50].

This article is organized as follows: section 2 describes the reference coupled problem in a compact form (details are provided in appendix A), while section 3 provides its two-scale formulation. Section 4 is dedicated to the validation of the proposed approach, on a sufficiently simple case that allows a reference solution to be computed, for comparison purpose. Finally, section 5 couples the previously built deterministic computational strategy with an uncertainty propagation scheme, namely the polynomial chaos expansion with collocation to provide a non-intrusive tool for stochastic analysis of a more complex-in-time loading case.

\section{Thermo-mechanical reference problem}

As a first step, we consider a weak thermo-mechanical coupling, only via dilatation effects. The influence of damage on thermal conductivity is therefore not taken into account. This can be justified by a not-so-high level of damage obtained; depending on the underlying physical nature of the damage (e.g. micro cracks) an higher value may be influent. On the other hand, as long as the mechanical nonlinearities are low, any adiabatic over-heating (internal heat generation with mechanical dissipation) can be neglected.

Phenomenological material modeling with internal variables are a practical tool for modeling material behavior. Usually, primal internal variables define the state of the material; the choice of a state potential allows the definition of the dual internal variables, via the state laws. For evolution laws, a pseudo potential is chosen. With partial Legendre-Fenchel transforms, the primal and dual internal variables can be interchanged. For the current case, the modeling choice is briefly recalled in the following.

We consider a quasi-static thermo-elasto-viscoplastic coupled model with damage, under small perturbations. The couples of dual variables are: heat flux and gradient of temperature $(q, \underline{Z})$, stress and elastic strain $\left(\sigma, \varepsilon^{e}\right)$ (the strain is partitioned into an elastic and a plastic part: $\varepsilon=\varepsilon^{e}+\varepsilon^{p}$ ), kinematic hardening variables $(\boldsymbol{\beta}, \boldsymbol{\alpha})$, isotropic hardening variables $(R, r)$, isotropic damage and associated energy release rate $\left(D, Y_{D}\right)$.

They are linked via state laws: $\underline{q}=-k \underline{Z}$ (Fourier's law), $\boldsymbol{\sigma}=\boldsymbol{E}(1-D)\left(\varepsilon^{e}-\varpi \theta \boldsymbol{\delta}\right)$ (Hooke's law 
with damage, and with the thermal dilatation coupling), and $Y=G(\boldsymbol{\sigma}, X)$ where

$$
X=\left[\begin{array}{c}
\boldsymbol{\beta} \\
R \\
D
\end{array}\right], \quad Y=\left[\begin{array}{c}
\boldsymbol{\alpha} \\
r \\
Y_{D}
\end{array}\right] .
$$

The evolution laws drive the evolution of the state of the material. They read

$$
\left[\begin{array}{c}
\dot{\varepsilon}^{p} \\
\dot{X}
\end{array}\right]=F\left(\left[\begin{array}{c}
\sigma \\
Y
\end{array}\right]\right)
$$

No evolution law pertain to the thermal part: since the thermal behavior is modeled as linear, there is no evolution of the state of the material.

These relations constitute the behavioral model of the material. The structural problem also involves conservation laws: the heat equation $\rho c \dot{\theta}=\operatorname{div} \underline{q}$, the equilibrium equation $\underline{\operatorname{div}} \boldsymbol{\sigma}+\underline{f}=0$, the thermal gradient and strain admissibility $\underline{Z}=\underline{\operatorname{grad}} \theta \underline{\text { and }} \varepsilon=\operatorname{grad} \underline{U}$, where $\theta$ is the temperature difference and $\underline{U}$ is the displacement field with respect to the reference state. To close the problem, an initial condition for the temperature, the initial state of internal variables, and boundary conditions have to be stated: a possible prescribed displacement $\underline{V}$, force $\underline{F}$, temperature $\theta_{d}$, thermal flux $w_{d}$ on parts of the boundary.

The damage model used herein is the one described in [34]. It is more deeply detailed in appendix A. Though embedding viscoplasticity, this model may suffer from mesh-dependency if localisation of damage and strain occurs. Since it is used herein up to not a high level of damage, this phenomena is not involved, therefore rendering this mode suited for the concerned application cases.

\section{Two-scale deterministic problem}

The fact that a two-time-scale model can be built is related to the possible description at two time scales of the prescribed quantities (boundary conditions, loads, etc.). They can be described as a slow time evolution along with rapid fluctuation in time, satisfying the pseudo-periodicity assumption: the evolution from one cycle to the other can be described at macroscopic scale.

We consider herein two time scales: one related to the overall smooth loading, with characteristic time $T$, and one related to the cycling loading, related to the period of a cycle $T_{c}$.

There are two main points in this approach (i) the fact that the solution fields are explicited with two separated time scales, and (ii) the pseudo-periodicity assumption on the fast time scale. The first direclty arises from the loadings: for the considered application cases, they exhibit such time scales. With a non-linear behavior, the pseudo-periodic assumption only holds in particular cases, where a single loading cycle only modifies slowly the internal state. Therefore, the cumulative effect can be captured by the long-time scale, and pseudo-periodicity is well satisfied as soon as the loadings exhibit also this property. This class of problem is considered in this submission, and a limitation of this study clearly resides in the fact that it can be applied to this specific situation. This situation is nevertheless a classical one, leading to fatigue analysis, cycle-jump techniques and so on.

\subsection{Thermal part}

We consider the loadings on the thermal problem (prescribed temperature, prescribed flux) as a superposition of two evolutions at the two aforementioned time scales:

$$
\theta_{d}=\theta_{d, M}+\theta_{d}^{\star} \quad \text { and } \quad w_{d}=w_{d, M}+w_{d}^{\star},
$$

where index $M$ stands for the macroscopic part (slow variation in time) and the superscript $\star$ stands for the microscopic part (fast variation in time, with quasi-periodic evolution from one micro cycle to the other, and a zero average on a cycle).

As a linear problem, the thermal problem can be split in two parts: a macro problem and a micro problem, and we superimpose the corresponding solutions. For instance, considering a prescribed temperature boundary condition only, the micro problem consists in finding $\theta^{\star}(t)$ on a time micro cycle $t \in\left[0, T_{c}\right]$ satisfying

$$
\begin{array}{r}
\rho c \dot{\theta}^{\star}=\operatorname{div}\left(-k \underline{\operatorname{grad}} \theta^{\star}\right) \\
\left.\theta^{\star}\right|_{\partial_{1} \Omega}=\theta_{d}^{\star}
\end{array}
$$

No specific initial condition is prescribed, since this micro problem is subjected to a periodicity condition on a fast cycle (periodic-in-time solution with period $T_{c}$ ), and we add a supplementary feature on the solution: a zero average in time. Indeed using the time average on a period

$$
<\bullet>=\frac{1}{T_{c}} \int_{0}^{T_{c}} \bullet d t
$$


the problem leads to

$$
\begin{aligned}
\rho c<\frac{\partial \theta^{\star}}{\partial t}>=\operatorname{div}(-k \underline{\operatorname{grad}} & \left.<\theta^{\star}>\right) \\
<\theta^{\star}>{ }_{\partial_{1} \Omega}= & <\theta_{d}^{\star}>
\end{aligned}
$$

by construction $\left.<\theta_{d}^{\star}\right\rangle=0$, with periodicity $\left\langle\frac{\partial \theta^{\star}}{\partial t}>=\frac{1}{T_{c}}\left[\theta^{\star}\left(T_{c}\right)-\theta^{\star}(0)\right]=0\right.$. So, if the problem is well-posed, it leads to

$$
<\theta^{\star}>=0 .
$$

To deal with periodicity constraint on the micro problem, different approaches can be used. The Fourier decomposition is not selected here, due to the potentially high number of modes required in the solution, depending on the spectral decomposition of the micro-loading. We therefore prefer a transient direct resolution on several micro-cycles, till a reasonably periodic solution is achieved (performed once for all due to the linearity of the thermal problem, and as soon as the shape of the micro-loading cycle $\theta_{d}^{\star}$ is unchanged). For the considered numerical cases, a maximum of 4 micro-cycles have to be computed that leads to a relative error in periodicity for $\theta^{\star}$, defined as

$$
\frac{\operatorname{mean}_{\text {spatial location }}\left|\theta^{\star}\left(T_{c}\right)-\theta^{\star}(0)\right|}{\max \left|\theta_{d}^{\star}\right|},
$$

to be less than $10^{-4}$.

\subsection{A generic two-scale description}

The scale ratio $\xi=T_{c} / T$ is assumed to be small. We first consider a representation of any quantity $\Phi$ with a two-time scale description: $\Phi(t, \tau)$ where $\tau=t / \xi$ is called the fast time variable. Separation of the scales relies on the assumption that $\xi$ is small, so that we may consider the functions of $\tau$ to be $T$-periodic. Finally, an asymptotic development with respect to $\xi[1]$ of the various quantities is derived as: $\Phi(t, \tau)=\Phi_{0}(t, \tau)+\xi \Phi_{1}(t, \tau)+\xi^{2} \Phi_{2}(t, \tau)+\cdots$ Indeed, the time derivative is now defined as: $\dot{\Phi}=\frac{\partial \Phi}{\partial t}+\frac{1}{\xi} \frac{\partial \Phi}{\partial \tau}$.

Note also that, due to the change in time variable, the previous average on a micro cycle is now denoted as $\left\langle\bullet>_{\tau}=\frac{1}{T_{c} / \xi} \int_{0}^{T_{c} / \xi} \bullet d \tau\right.$. The reference problem is then rewritten according to these expansions, and is now parametered with a small quantity $\xi$. The problem itself is then expanded according to different powers of $\xi$ leading to a succession of embedded sub-problems.

Classically, only the first orders of developments are taken into account.

For thermo-mechanical problems under concern, we consider that the time scale ratio $\xi$ is the same for both physics. Without loss of generality, we may consider that the two time scales are of the same order of magnitude; in this case, we only have $T \approx T_{c} / \xi$. In such a way, we end up with only one small parameter for the various asymptotic developments. If the time scale ratios, say $\xi$ and $\zeta$ were different in order of magnitude, we would need to hierarchically classify them, i.e. to determine the integers $j$ and $k$ so that $\xi^{j}=\zeta^{k}$, to be able to recover a problem with a single small parameter.

\subsection{Mechanical part}

Expanding the evolution laws with respect to the small parameter $\xi$ allows to identify the different orders of the development.

Since the loadings are given evolution functions, there is no need to state a particular approximation on them, except the fact that they exhibit two separated time scales. Therefore, we can give their expressions at order 0 only, i.e. $\underline{f}(t, \tau)=\underline{f}_{0}(t, \tau), \underline{F}(t, \tau)=\underline{F}_{0}(t, \tau)$ and $\underline{V}(t, \tau)=\underline{V}_{0}(t, \tau)$.

The same notations as for the thermal problem are used: the macroscopic time quantities will be denoted with a subscript $M$, and the microscopic corrections with a star.

Macroscopic quantities. Using order $\frac{1}{\xi}$ of the expansion for the evolution laws, we obtain

$$
\frac{\partial \varepsilon_{0}^{p}}{\partial \tau}=0, \frac{\partial X_{0}}{\partial \tau}=0
$$

so that

$$
\varepsilon_{M}^{p}=\varepsilon_{0}^{p}(t), X_{M}=X_{0}(t) .
$$

Using then order $\xi^{0}$ for the state laws, we obtain $Y_{0}=G\left(\sigma_{0}, X_{M}\right)$, and $Y_{M}=<Y_{0}>_{\tau}$. 
Residual quantities. Concerning the boundary conditions, the external forces and prescribed displacements may have components at both time scales. Nevertheless, for our development to hold, we restrict ourselves to cases where their eventual micro parts are quasi-periodic in time. If it is not the case, this will imply the equivalent of an edge effect in space (sort of an inner transient evolution) that cannot be resolved with an homogenization approach. For instance, with a load $\Phi_{0}(t, \tau)$, we define its macro part using the average on cycles as $\Phi_{M}(t)=<\Phi_{0}>_{\tau}$, and the residual as $\Phi^{\star}(t, \tau)=\Phi_{0}(t, \tau)-\Phi_{M}(t)$. As a consequence, we get $<\Phi^{\star}>_{\tau}=0$, which is consistent with its counterpart for thermal behavior (9).

With the previous results on macroscopic quantities, this also leads to $\varepsilon^{p, \star}=0$ and $X^{\star}=0$.

Macroscopic problem. The expansion of the admissibility conditions at order $\xi^{0}$ read: $\underline{\operatorname{div}} \boldsymbol{\sigma}_{0}+$ $\underline{f}=0$. The admissibility conditions at order $\xi^{0}$ therefore leads to the averaged (homogenized) equations:

- $\underline{\operatorname{div}}<\sigma_{0}>_{\tau}+\underline{f}_{M}=0$ and $<\sigma_{0}>_{\tau} \underline{n}=\underline{F}_{M}$ (on the corresponding boundary) which is the macroscopic equilibrium; therefore we define as the macroscopic stress $\boldsymbol{\sigma}_{M}:=<\boldsymbol{\sigma}_{0}>_{\tau}$,

- on the boundary with a prescribed displacement: $\underline{U}_{M}=<\underline{V}_{0}>_{\tau}$, and $\varepsilon_{M}=<\varepsilon_{0}>_{\tau}$.

With the previous results at order $\xi^{-1}$, the state laws at order $\xi^{0}$ are $\boldsymbol{\sigma}_{0}=\boldsymbol{E}\left(1-D_{M}\right)\left(\varepsilon_{0}^{e}-\right.$ $\left.\varpi \theta_{M} \boldsymbol{\delta}-\varpi \theta^{\star} \boldsymbol{\delta}\right)$, leading to

$$
\boldsymbol{\sigma}_{M}=\boldsymbol{E}\left(1-D_{M}\right)\left(\varepsilon_{M}^{e}-\varpi \theta_{M} \boldsymbol{\delta}\right),
$$

where $\boldsymbol{\varepsilon}_{M}^{e}(t):=<\boldsymbol{\varepsilon}_{0}^{e}>_{\tau}$.

Concerning the evolution laws, of the form $\dot{X}=F(\sigma, Y)$, the time derivative leads to the expansion at order $\xi^{0}$ as $\frac{\partial X_{0}}{\partial t}+\frac{\partial X_{1}}{\partial \tau}=F\left(\sigma_{0}, Y_{0}\right)$. The previous results at order $\xi^{-1}$ read $X_{0}(t)=X_{M}$. Together with the periodicity in $\tau$, the homogenized behavior is obtained by averaging

$$
\frac{\partial X_{M}}{\partial t}=<F\left(\boldsymbol{\sigma}_{M}+\boldsymbol{\sigma}^{\star}, Y_{0}\right)>_{\tau},
$$

where, using the previous results at order $\xi^{-1}$,

$$
Y_{0}=G\left(\boldsymbol{\sigma}_{M}+\boldsymbol{\sigma}^{\star}, X_{M}\right)
$$

and the last macro state laws are the post-processing

$$
Y_{M}=<Y_{0}>_{\tau}
$$

The macroscopic behavior therefore involves the coarsening of the behavior at the underlying scale, through the $\sigma^{\star}$ quantity. Its determination is given by the relocalisation step, giving the microscopic behavior.

Residual problem. The previous averaged part of the behavior at order $\xi^{0}$ has to be completed by the so-called residual terms, as

$$
\underline{\operatorname{div}} \boldsymbol{\sigma}^{\star}+\underline{f}^{\star}=0,
$$

for micro equilibrium, with boundary conditions $\sigma^{\star} \underline{n}=\underline{F}^{\star}, \underline{U}^{\star}=\underline{V}^{\star}$ (on the corresponding parts of the boundary). and with $\varepsilon^{\star}=\left[\operatorname{grad}\left(\underline{U}^{\star}\right)\right]_{\mathrm{sym}}$.

One may add the stress-strain relation as

$$
\boldsymbol{\sigma}^{\star}=\boldsymbol{E}\left(1-D_{M}\right)\left(\varepsilon^{e, \star}-\varpi \theta^{\star} \boldsymbol{\delta}\right),
$$

for closing the problem, since $\varepsilon^{e, \star}=\varepsilon^{\star}, \varepsilon^{p, \star}=0, X^{\star}=0$. It is remarkable that the mechanical residual problem is a static linear problem parametrized by $D_{M}$ field (and loaded with the known micro temperature field $\theta^{\star}$ ). Once solved, it provides the micro field $\boldsymbol{\sigma}^{\star}$, which is the required field to be able to express the macroscopic behavior (14),(15) and then (16). We can therefore consider that the complete macroscopic behavior is obtained by some phenomenological macroscopic equations coupled with a structural computation at micro scale.

\section{Numerical results and validation}

The pseudo-code corresponding to the proposed two-scale approach is described in appendix B. We consider here a 1D problem, of length $L$. The thermal problem consists in an initial zero temperature $\theta(x, t=0)=0$, a prescribed temperature to zero at left end point $(x=0)$, and a time-dependent prescribed temperature at right end point

$$
\theta(x=L, t, \tau)=\theta_{D} \sin (2 \pi t / T)+\theta_{d} \sin \left(2 \pi \tau / T_{c}\right) .
$$


Table 1: Problem parameters

\begin{tabular}{ll}
\hline Quantity & Value \\
\hline Structure length & $L=1 \mathrm{~m}$ \\
Studied time & $T=20000 \mathrm{~s}$ \\
Time scale ratio & $\zeta=10^{-3}$ \\
\hline Thermal capacity & $\rho c=4 \times 10^{6} \mathrm{~J}_{\mathrm{m}} \mathrm{m}^{-3} \cdot \mathrm{K}^{-1}$ \\
Thermal conductivity & $k=29 \mathrm{~W} / \mathrm{m} / \mathrm{K}$ \\
Thermal loading amplitude (macro) & $\theta_{D}=200{ }^{\circ} \mathrm{C}$ \\
Thermal loading amplitude (micro) & $\theta_{d}=80{ }^{\circ} \mathrm{C}$ \\
\hline Young modulus & $E=134 \mathrm{GPa}$ \\
Yield stress & $\sigma_{Y}=85 \mathrm{MPa}$ \\
Damage evolution modulus & $S=0.6 \mathrm{MPa}$ \\
Damage evolution exponent & $s=2$ \\
Linear kinematic hardening modulus & $Q=5400 \mathrm{MPa}$ \\
Saturation value for exponential isotropic hardening & $R_{\infty}=30 \mathrm{MPa}$ \\
Coefficient for exponential isotropic hardening & $\gamma=2$ \\
Norton law viscous modulus & $K_{v}=1220 \mathrm{MPa} \cdot \mathrm{s}^{1 / n_{v}}$ \\
Norton law viscous exponent & $n_{v}=2.5$ \\
Mechanical loading amplitude (macro) & $f_{D}=0.2 \mathrm{~N} / \mathrm{mm}^{3}$ \\
Mechanical loading amplitude (micro) & $f_{d}=0.08 \mathrm{~N} / \mathrm{mm}^{3}$ \\
\hline Lineic dilatation parameter & $\varpi=1.3 \times 10^{-5} \mathrm{~K}^{-1}$ \\
\hline
\end{tabular}

The mechanical problem is a non-freely dilatable structure where both end points are clamped, with an applied body force of (quasi-static) centrifugal type

$$
f(x, t, \tau)=f_{D} \sin (2 \pi t / T)+f_{d} \sin \left(2 \pi \tau / T_{c}\right) .
$$

The studied time interval is $T$ and the cycle period to time interval is $\xi=10^{-3}$, so $1 / \xi=10^{3}$ cycles are considered. In this case, it is still affordable to build a reference solution (with a single time scale, described with small time steps), for comparison purpose. Structural parameters are given in table 1, as well as the material parameters (Cr-Mo steel) that are detailed in appendix A.

A characteristic time for the transient thermal evolution is given as $\tau_{c}=(\rho c / k)(L / \pi)^{2}$. Since $T / \tau_{c} \approx 1.4$ the macroscopic thermal evolution is still in the transient regime, and so is the microscopic thermal evolution, for which the $1 \mathrm{D}$ thermal depth of penetration, see e.g. [36], for a load with period $T_{c}$, is $L_{c}=\sqrt{T_{c} / \tau_{c}} \times L / \pi^{3 / 2}$. Since $L_{c} / L \approx 0.7 \%$, the micro temperature is somehow localized at the boundary, but since the mechanical problem is also $1 \mathrm{D}$, its influence on the mechanical solution is not localized.

Concerning the regularization induced by the visco-plasticity, a characteristic time $\tau_{d}$ is more difficult to estimate due to the non-linearity; nevertheless, a dimensional analysis leads to $\tau_{d} \approx$ $K_{v}^{n_{v}} / E$, so $T / \tau_{d} \approx 1.6$ and $T_{c} / \tau_{d} \approx 0.16 \%$ so regularization is expected to happen, but though visco-plasticity induces a regularization, it does not prevent mesh dependency if localization occurs, see e.g. [49]. In the reported results, damage is sufficiently small to prevent this localization to occur. Extending the proposed approach to a mesh-independent damage localization model is a perspective to this article. But in any case, if such a localization happens, for higher levels of damage, it leads to an instability that entails the pseudo-periodic assumption (this can be seen as an "edge effect" localized in time). Detecting the occurrence of this may well be done with some error indicator in continuity between micro time cycles. To cope with it is easier than for the spatial edge effect: a switch between time integration schemes can be performed, before the instability occurs, the twotime-scale computation can be stopped, a classical mono-time-scale scheme (with short time steps) can be used during this phenomenon to pursue the computation, then a restart of the two-time-scale scheme can be performed. This will obviously fail if a succession of such events happens frequently in time, but in this case the problem is non-homogenizable in time.

Concerning spatial discretization, $n=200 \mathrm{P} 1$ finite elements (with a single integration point) are considered both for temperature and displacement. The macro time scale $[0, T]$ is discretized in $n_{M}=100$ time increments to cope with precision requirements for the macro part evolution only; the micro cycle being discretized with $n_{m}=50$ time increments. For the thermal problem the time integration scheme is implicit Euler, and for the mechanical problem, the non-linear equilibrium is solved using Newton iterations with a radial return algorithm for the constitutive relations. 


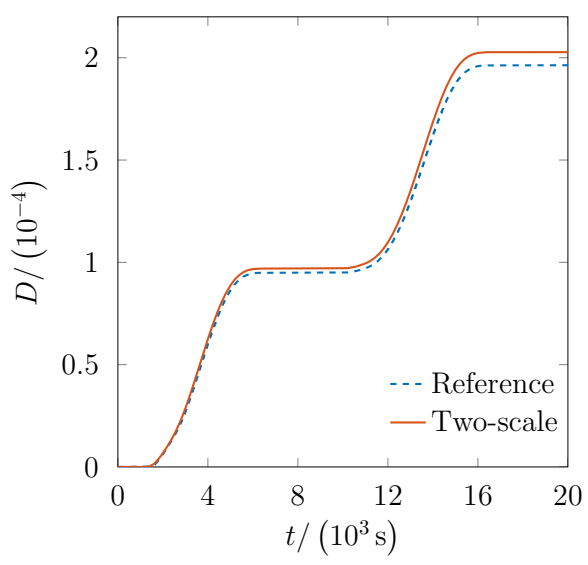

(a) Evolution of damage at the weakest Gauss point

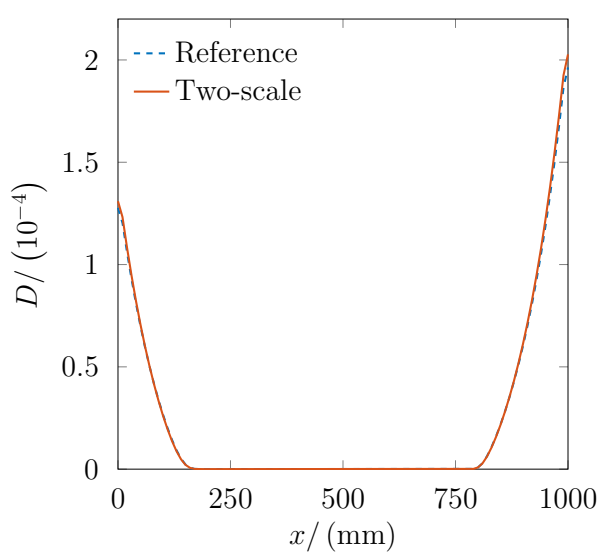

(b) Final damage field

Figure 1: Distribution of damage

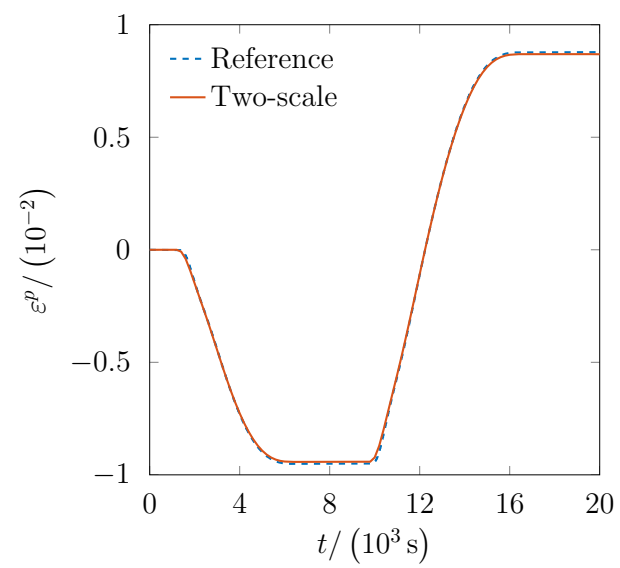

(a) Evolution of plastic strain at the weakest Gauss point

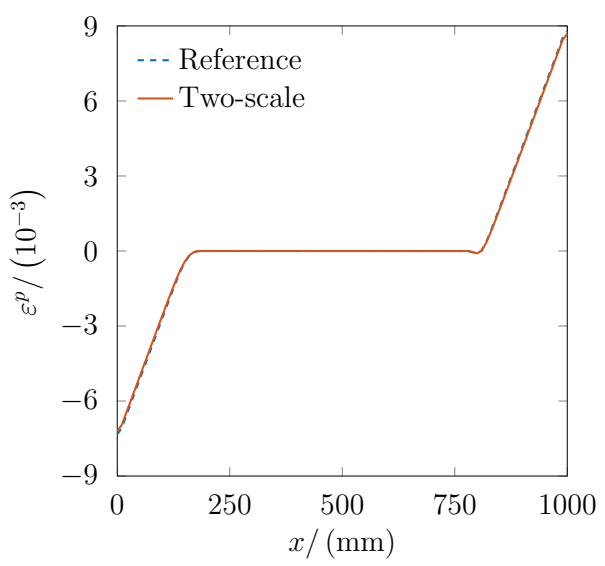

(b) Residual plastic strain

Figure 2: Distribution of plastic strain

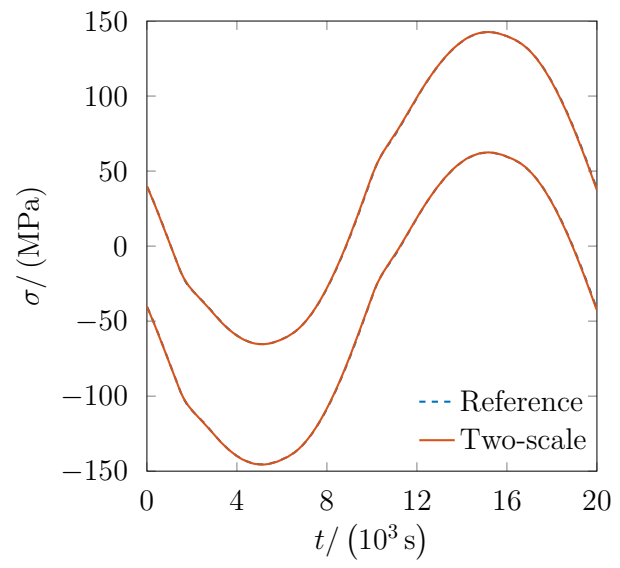

(a) Envelope of stress at the weakest Gauss point

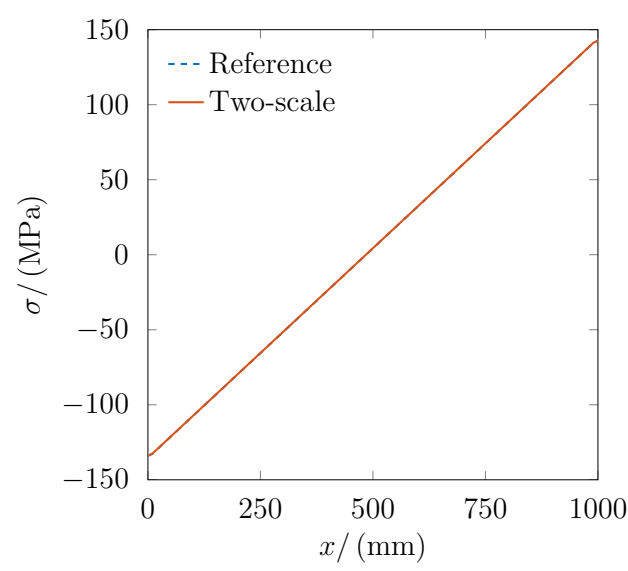

(b) Maximum stress distribution

Figure 3: Distribution of stress 


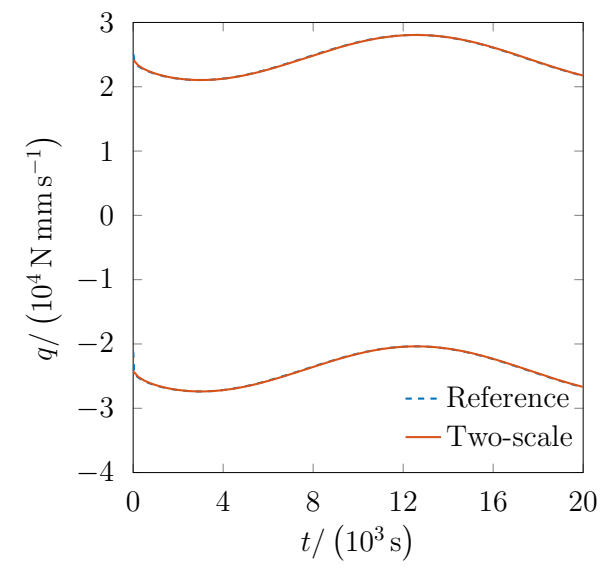

(a) Envelope of heat flux density at the weakest Gauss point

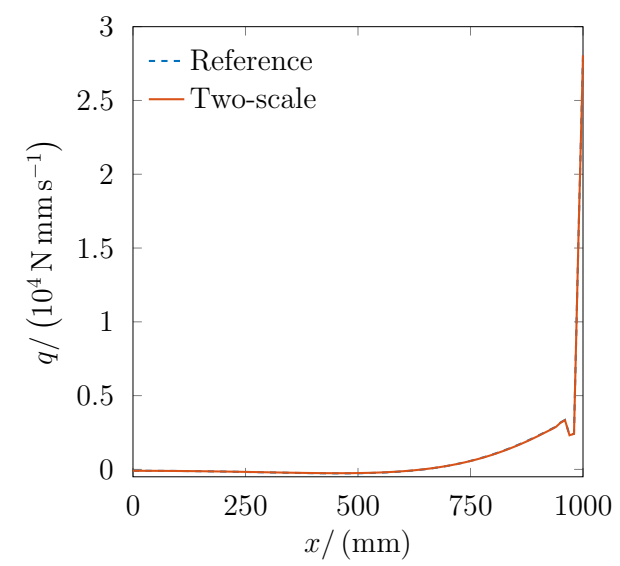

(b) Maximum heat flux density distribution

Figure 4: Distribution of heat flux density

Table 2: 2-norm errors on different space-time fields, between reference and two-time approximation

\begin{tabular}{ccccc}
\hline Quantity $\varrho$ & $D$ & $\sigma$ & $\beta$ & $\theta$ \\
Error $E r_{\varrho}$ & $2.7 \%$ & $0.78 \%$ & $1.8 \%$ & $1.3 \%$ \\
\hline
\end{tabular}

For comparison purpose, a reference single time scale computation would require $n_{m} / \xi$ time increments for both physics, while the two-time scale needs only $n_{m} \times n_{M}$ time increments for the mechanical part, and $n_{m}+n_{M}$ for the thermal part (due to linearity, the micro thermal cycle is precomputed once for all).

The error criteria to compare the multiscale solution with respect to the reference is

$$
E r_{\varrho}=\left(\frac{\int_{\Omega \times[0, T]}\left(\varrho_{r e f}-\varrho_{t s}\right) \cdot\left(\varrho_{r e f}-\varrho_{t s}\right) \mathrm{d} \Omega \mathrm{d} t}{\int_{\Omega \times[0, T]} \varrho_{r e f} \cdot \varrho_{r e f} \mathrm{~d} \Omega \mathrm{d} t}\right)^{1 / 2}
$$

with $\varrho=\{D, \sigma, \beta, \theta\}$. These errors are reported in Table 2 .

Concerning damage, an other comparison to the reference, computed herein at the macroscopic time steps $t$, allows to obtain the cumulative evolution in time of the error:

$$
e r_{D}(t)=\left(\frac{\int_{\Omega \times[0, t]}\left(D_{r e f}-D_{t s}\right) \cdot\left(D_{r e f}-D_{t s}\right) \mathrm{d} \Omega \mathrm{d} t^{\prime}}{\int_{\Omega \times[0, T]} D_{r e f} \cdot D_{r e f} \mathrm{~d} \Omega \mathrm{d} t^{\prime}}\right)^{1 / 2}
$$

Total number of global Newton-Raphson iterations is 0.0042 of the reference, and total number of iterations needed for the solution of non-linear mechanical behavior is 0.0282 of the reference. This illustrates the gain in computational cost of the two-scale approach with respect to the reference problem direct resolution. Nevertheless, this approach also embeds some approximations, but the values of the errors with respect to he reference are sufficiently small to consider it as a valuable numerical strategy, as illustrated with the comparisons on figures $1 \mathrm{a}$ and $1 \mathrm{~b}$. Note however, that as for any time evolution incremental schemes, the errors cumulated in time, as figure 5 illustrates it (note that the normalization of the error on this figure is related to the damage value on the somehow short time studied, which is also lower than for a larger time interval). Some error estimators would therefore be useful for checking purposes, and are still kept as perspectives of this study. Apart from the damage variable, comparisons of several other quantities are provided like the plastic strain (figure 2), the stress (figure 3). For the comparison of the thermal part, the heat flux density $(q)$ is calculated as post processing of the temperature field (figure 4).

\section{Uncertainty propagation}

Once a deterministic model is settled and validated, one can focus on the reliability of the values of its input parameters. Indeed, even a fine model relies on the precision of these values. To process a robust design, some uncertainties have to be taken into account. 


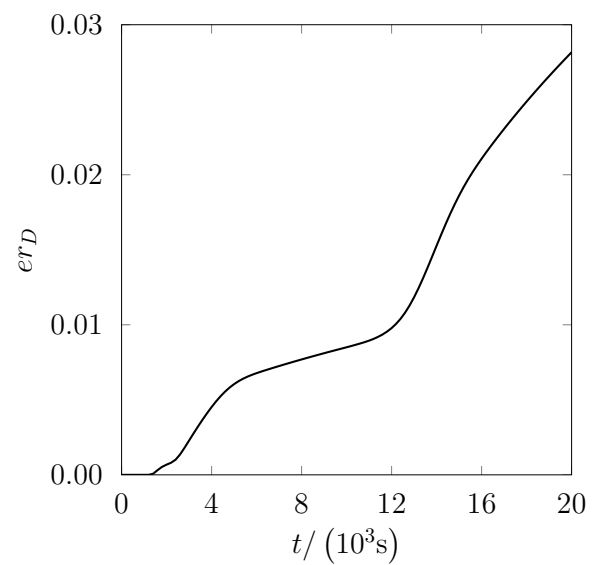

Figure 5: Evolution of $e r_{D}$

We consider herein the polynomial chaos expansion (PCE) $[22,16]$ with a stochastic collocation as a non-intrusive strategy to determine the stochastic model of the quantity of interest, as in [48, $26,25,50]$. Each input is expanded in chaoses with random variables following a chosen probability law. A minimization is done on the output expanded in polynomial chaoses that are calculated on collocation points, and gives the coefficients of the expansion.

Let $\boldsymbol{\zeta}$ denote the independent input random variable vector. We choose herein the damage field at the end of the studied time interval as the quantity of interest to analyze. It is expanded using a polynomial chaos as

$$
D(x, \boldsymbol{\zeta})=\sum_{i=0}^{\infty} D_{i}(x) \Psi_{i}(\boldsymbol{\zeta}) \approx \sum_{i=0}^{M-1} D_{i}(x) \Psi_{i}(\boldsymbol{\zeta}),
$$

where $D_{i}(x)$ are coefficients and $\Psi_{i}(\boldsymbol{\zeta})$ a multivariate polynomial basis. $M$ is the truncation threshold; classically, using all polynomials of degree less than or equal to $p$ leads to $M=(N+p) ! / N ! / p$ ! with $N$ being the number of input variables, defining the size of random vector $\zeta$. Then, a mean square fit is used to determine the unknown coefficients:

$$
D_{i}(x)=\underset{D_{i}(x)}{\operatorname{argmin}} \sum_{k=1}^{r}\left(D\left(x, \boldsymbol{\zeta}_{k}\right)-\sum_{i=0}^{M-1} D_{i}(x) \Psi_{i}\left(\boldsymbol{\zeta}_{k}\right)\right)^{2}
$$

where $r$ collocation points $\boldsymbol{\zeta}_{k}$ are used. To choose these points, a classical approach is to select the roots of the polynomial of order $p+1$, leading to $r=(p+1)^{N}$ (a Cartesian grid of points in the parametric space). Selecting a polynomial order $p$, there are $M$ unknown coefficients to find.

As for optimization, design and analysis of computer experiments, etc, non-intrusive uncertainty propagation is a many-query problem, for which each query requires a solve of a black-box model. Indeed, if this solution is computationally costly, these methods are rapidly intractable. This is why a surrogate cheap model is of interest for the black box. Nevertheless, building such a surrogate model also requires calls to the full model evaluation. Herein, we propose to use the two-scale model as such a surrogate model for the black box. Moreover, since it relies on homogenization, it is built without calls to the reference problem in an offline phase.

A more demanding problem is used in this section: the same 1D problem as for the validation test of section 4 is used, but the total simulation time is now multiplied by 300 , so that the macroscopic load does have 300 macroscopic cycles, the total number of micro-cycles (for a reference problem that would now be very time consuming) is therefore 300000 .

\subsection{Influence of a single random input}

To check the approach, and to proceed to a sensitivity analysis, the first step is usually to consider one input parameter as a random variable at a time, i.e. $N=1$. For the present case, we consider uncertainties arising from the loading amplitudes only, at each scale and for each physics, i.e. for $f_{D}, f_{d}, \theta_{D}$ and $\theta_{d}$. The uncertainty model consists in describing these inputs as random variables following a uniform probability distribution, whose bounds are given in Table 3.

For one random variable, $N=1$ and the random vector $\zeta$ is reduced to a random variable $\zeta$. If we denote with $\boldsymbol{d}$ the column vector storing the computed outputs $D\left(\zeta_{k}\right), \boldsymbol{\Psi}$ the matrix with known coefficients $\boldsymbol{\Psi}_{k j}=\Psi_{j}\left(\zeta_{k}\right)$, and $\boldsymbol{c}$ the column vector storing the unknown coefficients $D_{j}$, the minimization problem $(24)$ reads

$$
\left[\boldsymbol{\Psi}^{T} \boldsymbol{\Psi}\right] \boldsymbol{c}(x)=\boldsymbol{\Psi}^{T} \boldsymbol{d}(x) .
$$


Table 3: Random input parameters

\begin{tabular}{ll}
\hline$f_{D}$ & $0.2 \pm 0.02 \mathrm{~N} / \mathrm{mm}^{3}$ \\
$f_{d}$ & $0.08 \pm 0.01 \mathrm{~N} / \mathrm{mm}^{3}$ \\
$\theta_{D}$ & $200 \pm 50{ }^{\circ} \mathrm{C}$ \\
$\theta_{d}$ & $80 \pm 20{ }^{\circ} \mathrm{C}$ \\
\hline
\end{tabular}

Table 4: Comparison of cost and precision, depending on the PCE order

\begin{tabular}{ccccc}
\hline $\begin{array}{c}\text { PCE } \\
\text { order } p\end{array}$ & $\begin{array}{c}\text { No. unknown } \\
\text { coeff. } M\end{array}$ & $\begin{array}{c}\text { No. colloc. } \\
\text { points } r\end{array}$ & $e_{\mathrm{FIT}}$ & $e_{\mathrm{LOO}}$ \\
\hline 1 & 4 & 8 & $4.1 \%$ & $8.1 \%$ \\
2 & 10 & 27 & $1.2 \%$ & $2.4 \%$ \\
3 & 20 & 64 & $0.54 \%$ & $0.93 \%$ \\
4 & 35 & 125 & $0.34 \%$ & $0.60 \%$ \\
\hline
\end{tabular}

For obtaining these results, the expansion order has been a priori selected to be $p=2$.

Figures 6 and 7 provide a post-treatment of the stochastic model of the final damage field (with its mean value, envelopes, and standard deviation).

In the chaos expansion, Legendre polynomials are herein selected because they are orthogonal with respect to the uniform probability density measure.

All input random variables have same order of magnitude influence on the dispersion of the value of the damage field, except for the micro thermal loading $\theta_{d}$ that has a much less influence.

\subsection{Influence of combined random inputs}

With the previous results, we can now model the dependencies of combined loading random inputs $\left(f_{D}, f_{d}, \theta_{D}\right)$. Indeed, the micro-thermal loading has the weakest effect, so it has been discarded from the set of $N=3$ influential parameters in the following study.

Minimization problem given by equation (25) for a random variable can be generalized easily with a random vector $\zeta$ by considering components $\zeta_{i}$ of this vector. Since PCE coefficients are fitted with a least square minimization, they minimize the fit error $\boldsymbol{e}(x)=\boldsymbol{d}(x)-\boldsymbol{\Psi} \boldsymbol{c}(x)$. A post-processing fit error can be built as

$$
e_{\mathrm{FIT}}=\max _{x} e(x) \quad \text { with } \quad e(x)=\frac{\|\boldsymbol{e}(x)\|_{\infty}}{\max _{x}\|\boldsymbol{d}(x)\|_{\infty}}
$$

Depending on the PCE order $p$, this fit error may vary. This error has to be combined with the analysis cost, mainly driven by the number of deterministic runs (i.e. the number $r$ of collocation points). With the previous rule for choosing the collocation points, the obtained results are summarized in Table 4. Another costless error indicator is based on the leave-one-out (LOO) crossvalidation approach [8]. This involves getting rid of one collocation point at a time, the corresponding slightly reduced set of outputs is then used to fit a new set of coefficients. The interpolation is then performed to recompute the approximation at the discarded collocation point. The same error $e_{\mathrm{FIT}}$ is then computed each time, and the LOO indicator $e_{\mathrm{LOO}}$ is the maximum value of these errors. It therefore belongs to a de-refinement error indicator category. Table 4 also reports its values. These results show the convergence of the approach, and are also an indication on the choice of truncation for the PCE; in this case, we select a polynomial order 3. The non-linear nature of the problem is illustrated by the fact that otherwise, a linear metamodel, i.e. PCE of order $p=1$ would be sufficient.

Concerning the optimality of the truncation, an illustration can be made by post-treatment. If PCE metamodels up to order $p$ are built, each using its own set of $r(p)$ collocation points, where the values of the quantity of interest are available, fit and LOO errors can be computed on the cumulative set of distinct collocations points for orders less than or equal to $p$. Table 5 reports these results; we notice that the indicators are quite stable, so that each independent order is close to the optimum. This is different from the convergence of the minimum least square when increasing the order $p$ on a fixed set of collocation points. If we check the fit error of the previously built PCE models on the full set of collocation points, we indeed exhibit the convergence reported in Table 6 .

Figure 8 shows the influence of the three random variables. Integrating the three influences, the variation is then larger than for each random variable but stays in the same range with keeping the same shape. 

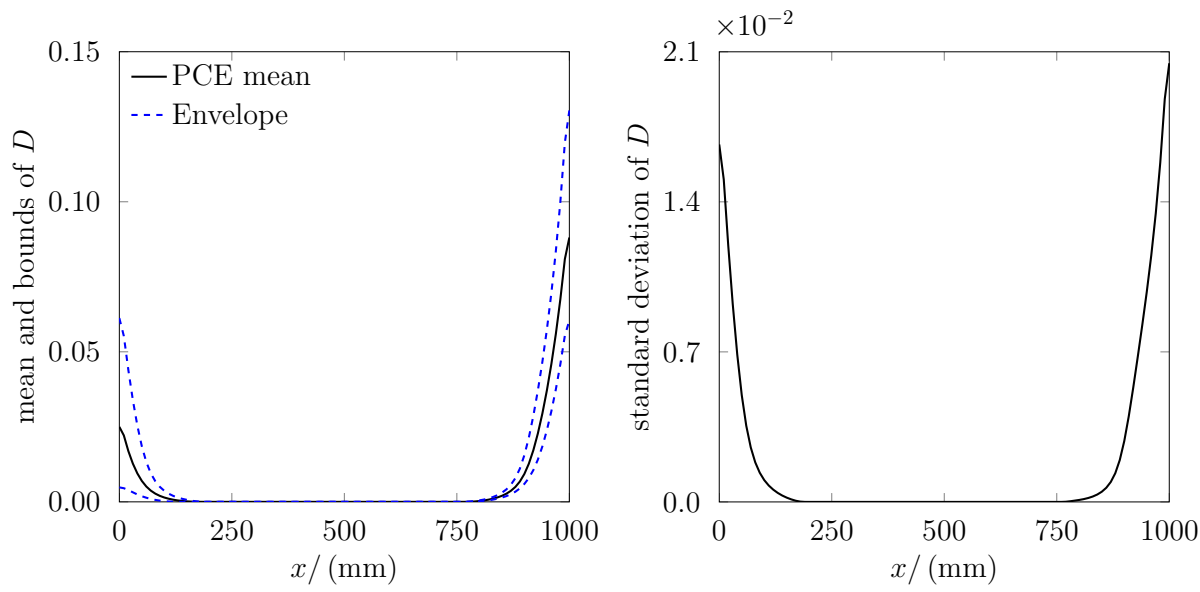

(a) Macro-load amplitude $f_{D}$
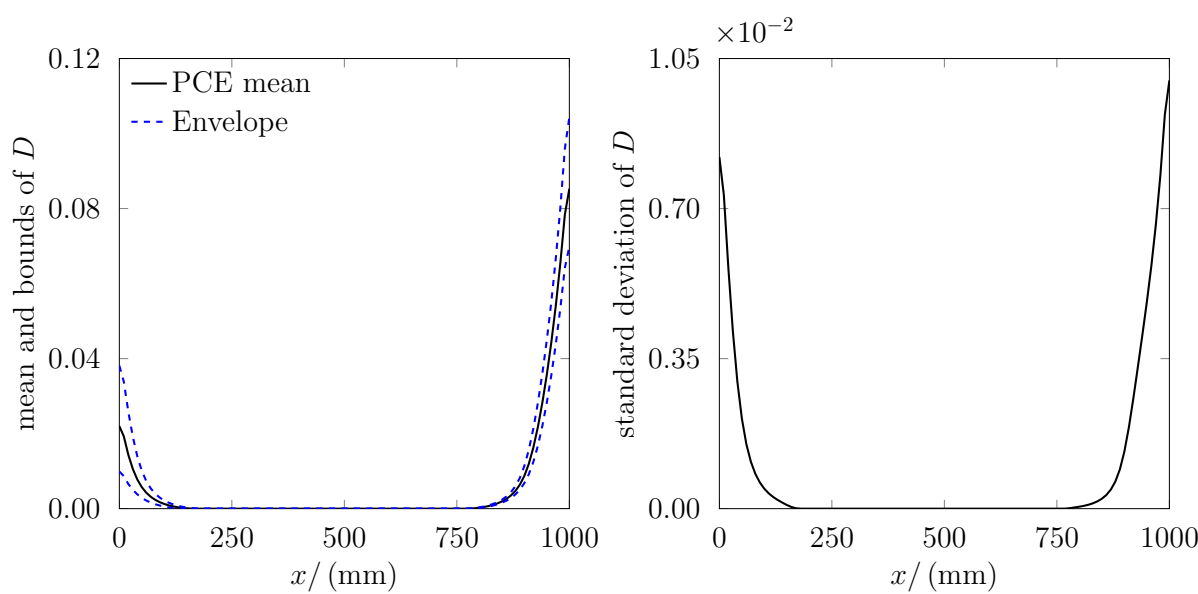

(b) Micro-load amplitude $f_{d}$

Figure 6: Influence of the mechanical load amplitudes on the final damage field

Table 5: Verification on the cumulative set of collocation points

\begin{tabular}{cccc}
\hline $\begin{array}{c}\text { PCE } \\
\text { order } p\end{array}$ & $\begin{array}{c}\text { cumulative number } \\
\text { of colloc. points }\end{array}$ & $e_{\mathrm{FIT}}$ & $e_{\mathrm{LOO}}$ \\
\hline 2 & 35 & $1.2 \%$ & $2.4 \%$ \\
3 & 99 & $0.54 \%$ & $0.86 \%$ \\
4 & 223 & $0.34 \%$ & $0.51 \%$ \\
\hline
\end{tabular}

Table 6: Convergence on a fixed set of 223 verification points

\begin{tabular}{ccc}
\hline $\begin{array}{c}\text { PCE } \\
\text { order } p\end{array}$ & $\begin{array}{c}\text { cumulative number } \\
\text { of colloc. points }\end{array}$ & $e_{\text {FIT }}$ \\
\hline 1 & 223 & $14 \%$ \\
2 & 223 & $3.4 \%$ \\
3 & 223 & $0.71 \%$ \\
4 & 223 & $0.34 \%$ \\
\hline
\end{tabular}



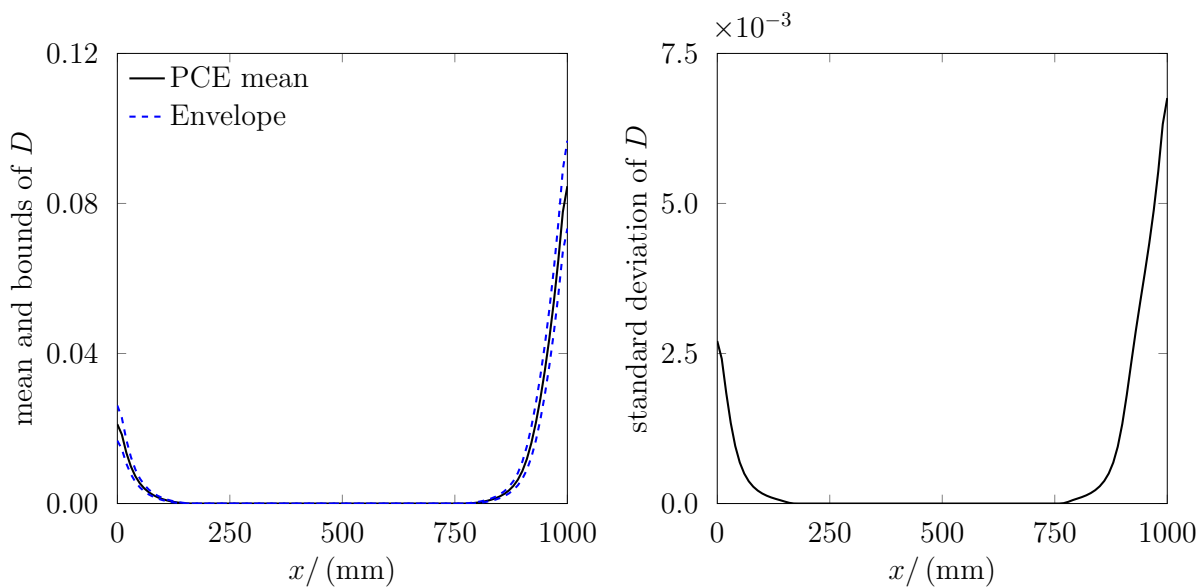

(a) Macro-load amplitude $\theta_{D}$
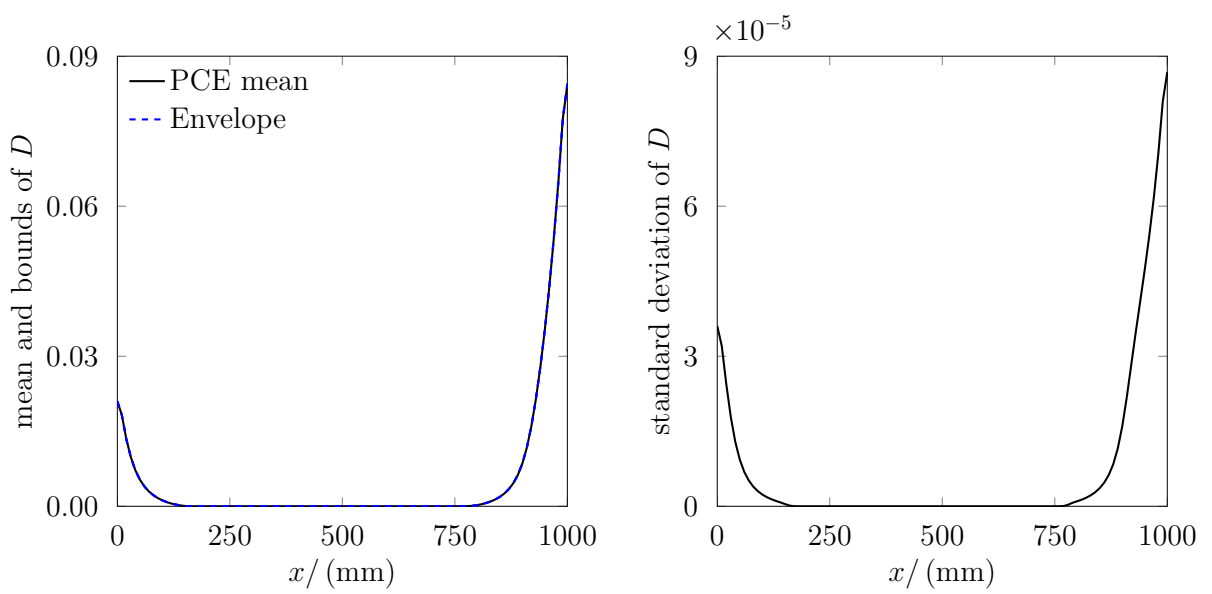

(b) Micro-load amplitude $\theta_{d}$

Figure 7: Influence of the thermal load amplitudes on the final damage field
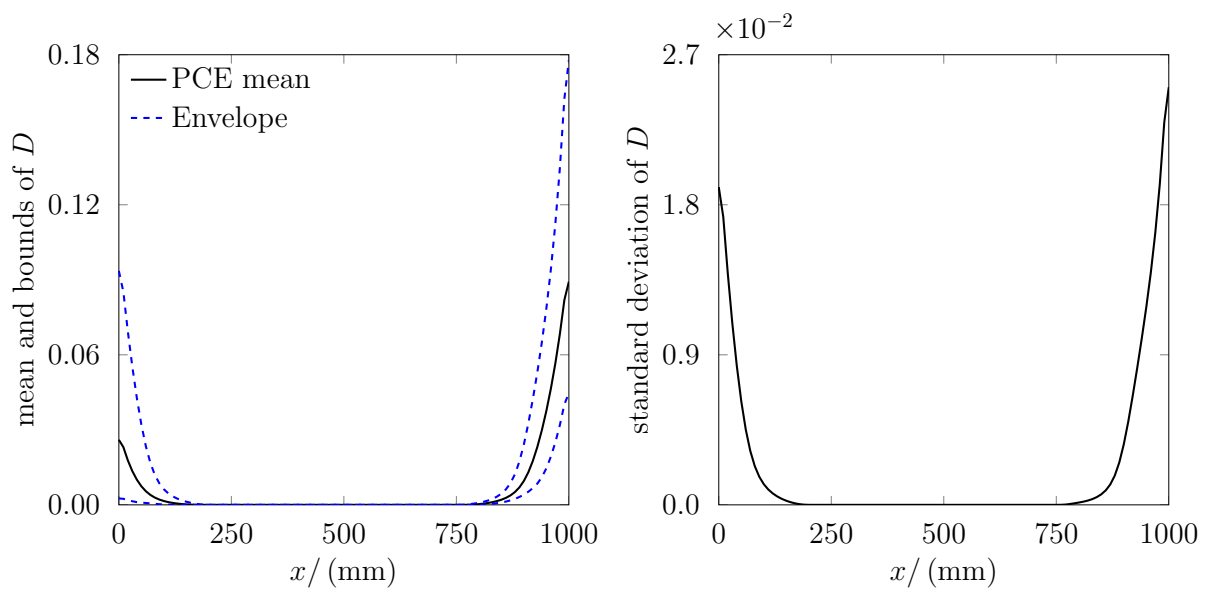

Figure 8: Combined influences of $f_{D}, f_{d}$ and $\theta_{D}$ on the final damage field 
Once the stochastic metamodel based on the PCE has been settled, it can be used to analyze at low cost the structure, submitted to random inputs. For instance structural reliability analysis [30], limit state determination when damage reach a critical value, failure probability if operated beyond the safe parametric domain.

\section{Conclusions}

In this article, we dealt with the case of fatigue mechanism that develops during cyclic loading of mechanical parts, using a thermal coupled elasto-visco-plastic model with damage. Though the examples are restricted to $1 \mathrm{D}$ cases, large number of pseudo-periodic cycling loadings are tackled using a time homogenization strategy. Uncertainty quantification is also embedded, using a nonintrusive polynomial chaos expansion (PCE) with collocation. Using surrogate models, arising from homogenization and PCE, the computing cost remain affordable.

As for perspectives, when addressing 3D problems with heterogeneous composite structures, one can also consider embedding a spatial homogenization, as in [51], to keep the cost of a single deterministic run affordable. Increasing the number of random parameters also drastically increases the cost of the overall analysis, so sparse approaches can be considered as well (e.g. sparse PCE, as in [5]). For these approaches also, as mentioned previously, some error estimators would be welcome for checking and confidence issues.

Extension to other damage models is also of interest.

Acknowledgements. The authors wish to thank Carnot Institute Ingénierie@Lyon (MURMUR project) and the China Scholarship Council (CSC grant 201801810065) for grants.

\section{A Detailed forms of the state laws and evolution laws}

The considered constitutive relations are [34]:

- State laws

$$
\begin{aligned}
\boldsymbol{\sigma} & =\boldsymbol{E}(1-D)\left(\boldsymbol{\varepsilon}^{e}-\varpi \theta \boldsymbol{\delta}\right) \\
\boldsymbol{\beta} & =\boldsymbol{C} \boldsymbol{\alpha} \\
R & =R_{\infty}(1-\exp (-\gamma r)) \\
Y_{D} & =\frac{\tilde{\sigma}_{e q}^{2} R_{v}}{2 E}
\end{aligned}
$$

$\boldsymbol{E}$ is the Hooke's tensor which is a function of the modulus of elasticity $E$ and Poisson's ratio $\nu . C$ is a tensor containing material parameter $C$ that describes kinematic hardening. $R_{\infty}$ and $\gamma$ are material parameters describing isotropic hardening. The triaxiality function $R_{v}=$ $\frac{2}{3}(1+\nu)+3(1-2 \nu)\left(\frac{\sigma_{h}}{\sigma_{e q}}\right)^{2}$, where $\sigma_{h}=\frac{1}{3} \operatorname{Tr}(\boldsymbol{\sigma})$ represents the hydrostatic part of the stress tensor, $\sigma_{e q}$ is the von Mises equivalent stress defined as $\sigma_{e q}=\sqrt{\frac{3}{2} \boldsymbol{\sigma}^{D}: \boldsymbol{\sigma}^{D}}$ with $\boldsymbol{\sigma}^{D}=\boldsymbol{\sigma}-\sigma_{h} \boldsymbol{\delta}$ being the deviatoric stress. The ratio $\frac{\sigma_{h}}{\sigma_{e q}}$ in the definition of $R_{v}$ is called the triaxiality ratio and $\tilde{\sigma}_{e q}$ is the equivalent of the effective stress defined as $\tilde{\sigma}_{e q}=\sqrt{\frac{3}{2} \frac{\boldsymbol{\sigma}^{D}}{(1-D)}: \frac{\boldsymbol{\sigma}^{D}}{(1-D)}}$.

- Evolution equations

$$
\begin{aligned}
\dot{\boldsymbol{\varepsilon}}^{p} & =\frac{\partial f^{p}}{\partial \boldsymbol{\sigma}}\left\langle\frac{f_{p}}{K_{v}}\right\rangle^{n_{v}}, \\
\dot{\boldsymbol{\alpha}} & =-\frac{\partial f^{p}}{\partial \boldsymbol{\beta}}\left\langle\frac{f_{p}}{K_{v}}\right\rangle^{n_{v}}, \\
\dot{r} & =-\frac{\partial f^{p}}{\partial R}\left\langle\frac{f_{p}}{K_{v}}\right\rangle^{n_{v}}, \\
\dot{D} & =\left(\frac{Y_{D}}{S}\right)^{s} \frac{1}{1-D}\left\langle\frac{f_{p}}{K_{v}}\right\rangle^{n_{v}},
\end{aligned}
$$

with the von Mises yield function $f^{p}$ delimiting the elastic domain and is defined as

$$
f^{p}=\sqrt{\frac{3}{2}\left[\left(\frac{\boldsymbol{\sigma}^{D}}{1-D}-\boldsymbol{\beta}\right):\left(\frac{\boldsymbol{\sigma}^{D}}{1-D}-\boldsymbol{\beta}\right)\right]}-R-\sigma_{y},
$$

where $\sigma_{y}$ is the yield stress. The viscous coefficient $K_{v}$ and viscous exponent $n_{v}$ are material parameters. The material parameters $S$ and $s$ describe the damage evolution. 


\section{B Resolution algorithm}

The pseudo-code of algorithm 1 is used to solve the two-time-scale problem under concerns. It uses an incremental scheme on the macro time steps, embedding a single Newton-like loop. Inside this last loop, computations are performed on the current micro-time cycle (behind the considered macro time step).

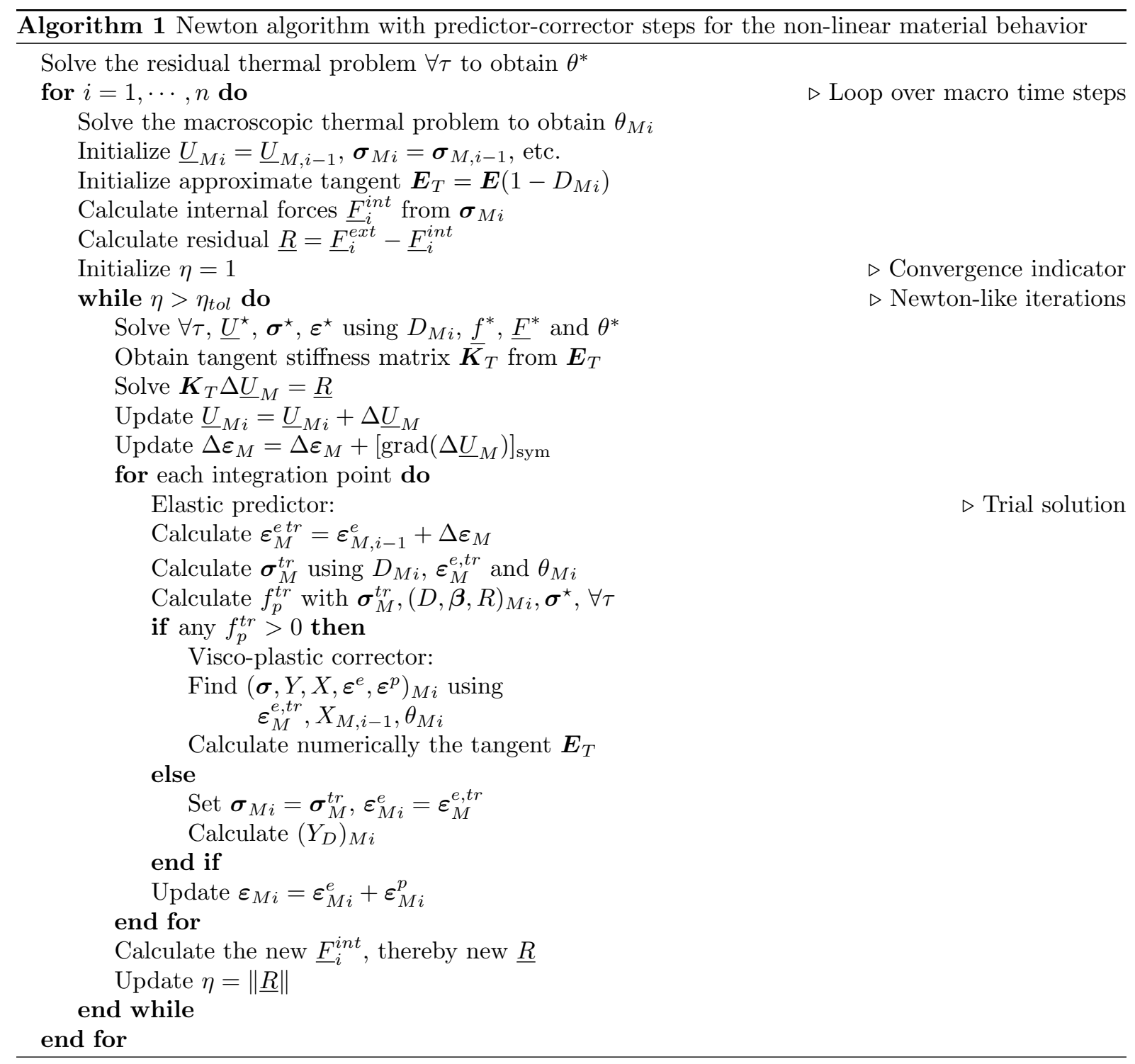

\section{References}

[1] A. Bensoussan, J.-L. Lions, and G. Papanicolaou. Asymptotic Analysis for Periodic Structures. North Holland, Amsterdam, 1978.

[2] Mainak Bhattacharyya, David Dureisseix, and Beatrice Faverjon. A unified approach based on temporal homogenisation and cycle jump for thermo-mechanical combined cycle fatigue. International Journal of Fatigue, 131:105320, 2020. doi:10.1016/j.ijfatigue.2019.105320.

[3] Mainak Bhattacharyya, Amélie Fau, Rodrigue Desmorat, Shadi Alameddin, David Néron, Pierre Ladevèze, and Udo Nackenhorst. A kinetic two-scale damage model for high-cycle fatigue simulation using multi-temporal LATIN framework. European Journal of Mechanics - A/Solids, 77:103808, sep 2019. doi:10.1016/j . euromechsol.2019.103808.

[4] Mainak Bhattacharyya, Amelie Fau, Udo Nackenhorst, David Néron, and Pierre Ladevèze. A LATIN-based model reduction approach for the simulation of cycling damage. Computational Mechanics, 62(4):725-743, 2018. doi:10.1007/s00466-017-1523-z. 
[5] G. Blatman and B. Sudret. Adaptive sparse polynomial chaos expansion based on least angle regression. Journal of Computational Physics, 230(6):2345-2367, 2011. doi:10.1016/j.jcp. 2010.12 .021$.

[6] Didier Bodin, Gilles Pijaudier-Cabot, Chantal de La Roche, Jean-Michel Piau, and Armelle Chabot. Continuum damage approach to asphalt concrete fatigue modeling. Journal of Engineering Mechanics, 130(6):700-708, 2004. doi:10.1061/(ASCE) 0733-9399(2004)130:6(700).

[7] C. Boutin and H. Wong. Study of thermosensitive heterogeneous media via space-time homogenisation. European Journal of Mechanics A/Solids, 17(6):939-968, 1998. doi:10.1016/ S0997-7538(98) 90503-4.

[8] Olivier Chapelle, Vladimir Vapnik, and Yoshua Bengio. Model selection for small sample regression. Machine Learning, 48(1-3):9-23, 2002. doi:10.1023/a:1013943418833.

[9] C. L. Chow and Y. Wei. A model of continuum damage mechanics for fatigue failure. International Journal of Fracture, 50(4):301-306, 8 1991. doi:10.1007/BF00032199.

[10] J.-Y. Cognard and P. Ladevèze. A large time increment approach for cyclic plasticity. International Journal of Plasticity, 9(2):141-157, 1993. doi:10.1016/0749-6419(93) 90026-M.

[11] D. Cojocaru and A. M. Karlsson. A simple numerical method of cycle jumps for cyclically loaded structures. International Journal of Fatigue, 28(12):1677-1689, 2006. doi:10.1016/j. ijfatigue.2006.01.010.

[12] A. Combescure, A. Gravouil, and B. Herry. An algorithm to solve transient structural non-linear problems for non-matching time-space domains. Computers and Structures, 81(12):1211-1222, 2003. doi:10.1016/S0045-7949(03)00037-3.

[13] Anne Devulder, Denis Aubry, and Guillaume Puel. Two-time scale fatigue modelling: application to damage. Computational Mechanics, 45(6):637-646, 2010. doi:10.1007/ s00466-010-0476-2.

[14] Charbel Farhat and Marion Chandesris. Time-decomposed parallel time-integrators: theory and feasibility studies for fluid, structure, and fluid-structure applications. International Journal for Numerical Methods in Engineering, 58(9):1397-1434, 2003. doi:10.1002/nme.860.

[15] A. Fatemi and L. Yang. Cumulative fatigue damage and life prediction theories: a survey of the start of the art for homogeneous materials. International Journal of Fatigue, 20(1):9-34, 1998. doi:10.1016/S0142-1123(97)00081-9.

[16] Béatrice Faverjon and Roger Ghanem. Stochastic inversion in acoustic scattering. The Journal of the Acoustical Society of America, 119(6):3577-3588, 2006. doi:10.1121/1.2200149.

[17] Fatima-Ezzahra Fekak, Michael Brun, Anthony Gravouil, and Bruno Depale. A new heterogeneous asynchronous explicit-implicit time integrator for nonsmooth dynamics. Computational Mechanics, 60(1):1-21, 3 2017. doi:10.1007/s00466-017-1397-0.

[18] Frédéric Feyel. A multilevel finite element method $\left(\mathrm{FE}^{2}\right)$ to describe the response of highly non-linear structures using generalized continua. Computer Methods in Applied Mechanics and Engineering, 192(28-30):3233-3244, 2003. doi:10.1016/S0045-7825(03)00348-7.

[19] Frédéric Feyel and Jean-Louis Chaboche. $\mathrm{FE}^{2}$ multiscale approach for modelling the elastoviscoplastic behaviour of long fiber $\mathrm{SiC} / \mathrm{Ti}$ composite materials. Computer Methods in Applied Mechanics and Engineering, 183(3-4):309-330, 2000. doi:10.1016/S0045-7825(99)00224-8.

[20] Jacob Fish, Mahesh Bailakanavar, Lynn Powers, and Thomas Cook. Multiscale fatigue life prediction model for heterogeneous materials. International Journal for Numerical Methods in Engineering, 91(10):1087-1104, aug 2012. doi:10.1002/nme.4307.

[21] Jacob Fish and Caglar Oskay. A nonlocal multiscale fatigue model. Mechanics of Advanced Materials and Structures, 12(6):485-500, 2005. doi:10.1080/15376490500259319.

[22] R. G. Ghanem and P. D. Spanos. Stochastic Finite Elements: A Spectral Approach. SpringerVerlag, New York, 1991.

[23] T. Guennouni. On a computational method for cycling loading: the time homogenization. Mathematical Modelling and Numerical Analysis, 22(3):417-455, 1988. In french.

[24] T. Guennouni and D. Aubry. Réponse homogénéisée en temps de structures sous chargements cycliques. Comptes rendus de l'Académie des sciences. Série 2, Mécanique, Physique, Chimie, Sciences de l'univers, Sciences de la Terre, 303(20):1765-1768, 1986. In French.

[25] Shuping Huang, Sankaran Mahadevan, and Ramesh Rebba. Collocation-based stochastic finite element analysis for random field problems. Probabilistic Engineering Mechanics, 22(2):194-205, 2007. doi:10.1016/j.probengmech.2006.11.004. 
[26] S. S. Isukapalli, A. Roy, and P. G. Georgopoulos. Stochastic response surface methods (SRSMs) for uncertainty propagation: Application to environmental and biological systems. Risk Analysis, 18(3):351-363, jun 1998. doi:10.1111/j.1539-6924.1998.tb01301.x.

[27] Franck Jourdan. Numerical wear modeling in dynamics and large strains: Application to knee joint prostheses. Wear, 261(3-4):283-292, 2006. doi:10.1016/j . wear.2005.10.007.

[28] S. Kruch. Numerical analysis of an engine turbine disk loaded with a large number of thermomechanical cycles. In D. R. J. Owen, E. Oñate, and E. Hinton, editors, 3rd International Conference on Computational Plasticity, Barcelone, 1992. Pineridge Press. Swansea, U.K.

[29] Pierre Ladevèze and Anthony Nouy. On a multiscale computational strategy with time and space homogenization for structural mechanics. Computer Methods in Applied Mechanics and Engineering, 192(28-30):3061-3087, 2003. doi:10.1016/S0045-7825(03)00341-4.

[30] Maurice Lemaire, Alaa Chateauneuf, and Jean-Claude Mitteau. Structural Reliability. ISTE, jan 2009. doi:10.1002/9780470611708.

[31] J. Lemaitre, J.-P. Sermage, and R. Desmorat. A two scale damage concept applied to fatigue. International Journal of Fracture, 97(1-4):67-81, 1999. doi:10.1023/A:1018641414428.

[32] Jean Lemaitre. A Course on Damage Mechanics. Springer-Verlag, Wien-New York, 1996.

[33] Jean Lemaitre and Jean-Louis Chaboche. Mechanics of Solid Materials. Cambridge University Press, 1994. doi:10.1017/CB09781139167970.

[34] Jean Lemaitre and Rodrigue Desmorat. Engineering Damage Mechanics. Springer-Verlag, 2005. doi:10.1007/b138882.

[35] P. M. Lesne and S. Savalle. An efficient cycles jump technique for viscoplastic structure calculations involving large number of cycles. In D. R. J. Owen, E. Hinton, and E. Oñate, editors, 2nd International Conference on Computational Plasticity, pages 591-602, Barcelona, Spain, 1989. Pineridge Press, Swansea, U.K.

[36] J.H. Lienhard, IV and J.H. Lienhard, V. A Heat Transfer Textbook. Dover Publications, Mineola, NY, 5th edition, 2019.

[37] Yvon Maday and Gabriel Turinici. The parareal in time iterative solver: a further direction to parallel implementation. In Domain Decomposition Methods in Science and Engineering. Lecture Notes in Computational Science and Engineering, volume 40, pages 441-448. Springer-Verlag, 2005. doi:10.1007/3-540-26825-1_45.

[38] J. J. Madge, S. B. Leen, I. R. McColl, and P. H. Shipway. Contact-evolution based prediction of fretting fatigue life: Effect of slip amplitude. Wear, 262(9-10):1159-1170, 2007. doi:10.1016/ j.wear.2006.11.004.

[39] E. Malachanne, F. Jourdan, and D. Dureisseix. Numerical model of bone remodeling sensitive to loading frequency through a poroelastic behavior and internal fluid movements. Journal of the Mechanical Behavior of Biomedical Materials, 4(6):849-857, 2011. MSC2010: 92C10, 76S99, 74F10. doi:10.1016/j.jmbbm.2011.03.004.

[40] David Néron and David Dureisseix. A computational strategy for thermo-poroelastic structures with a time-space interface coupling. International Journal for Numerical Methods in Engineering, 75(9):1053-1084, 2008. doi:10.1002/nme.2283.

[41] Caglar Oskay and Jacob Fish. Fatigue life prediction using 2-scale temporal asymptotic homogenization. International Journal for Numerical Methods in Engineering, 61(3):329-359, sep 2004. doi:10.1002/nme.1069.

[42] Caglar Oskay and Jacob Fish. Multiscale modeling of fatigue for ductile materials. International Journal for Multiscale Computational Engineering, 2(3):329-354, 2004. doi:10.1615/ intjmultcompeng.v2.i3.10.

[43] Guillaume Puel and D. Aubry. Efficient fatigue simulation using periodic homogenization with multiple time scales. International Journal for Multiscale Computational Engineering, 12(4):291-318, 2014. doi:10.1615/intjmultcompeng. 2014010036.

[44] Guillaume Puel and Denis Aubry. Material fatigue simulation using a periodic timehomogenisation method. European Journal of Computational Mechanics, 21(3-6):312-324, 2012. doi:10.1080/17797179.2012.714853.

[45] G. Saussine, C. Cholet, P.-E. Gautier, F. Dubois, C. Bohatier, and J. J. Moreau. Modelling ballast behaviour under dynamic loading, part 1: A 2D polygonal discrete element method approach. Computer Methods in Applied Mechanics and Engineering, 195(19-22):2841-2859, 2006. doi:10.1016/j.cma.2005.07.006. 
[46] Akke S. J. Suiker and René de Borst. A numerical model for the cyclic deterioration of railway tracks. International Journal for Numerical Methods in Engineering, 57:441-470, 2003. doi: 10.1002/nme.683.

[47] Ahmad Takash, Marianne Beringhier, Mohammad Hammoud, and Jean-Claude Grandidier. On the validation of the Proper Generalized Decomposition method with finite element method: 3D heat problem under cyclic loading. In Mechanism, Machine, Robotics and Mechatronics Sciences, pages 3-13. Springer International Publishing, 2018. doi:10.1007/978-3-319-89911-4_1.

[48] Menner A. Tatang, Wenwei Pan, Ronald G. Prinn, and Gregory J. McRae. An efficient method for parametric uncertainty analysis of numerical geophysical models. Journal of Geophysical Research: Atmospheres, 102(D18):21925-21932, sep 1997. doi:10.1029/97jd01654.

[49] W. M. Wang and L. J. Sluys. Formulation of an implicit algorithm for finite deformation viscoplasticity. International Journal of Solids and Structures, 37(48-50):7329-7348, nov 2000. doi:10.1016/s0020-7683(00)00201-8.

[50] J. Yang, B. Faverjon, D. Dureisseix, P. Swider, S. Marburg, H. Peters, and N. Kessissoglou. Prediction of the intramembranous tissue formation during perisprosthetic healing with uncertainties. Part 2. Global clinical healing due to combination of random sources. Computer Methods in Biomechanics and Biomedical Engineering, 19(13):1387-1394, 2016. doi: 10.1080/10255842.2016.1143465.

[51] Zhiqiang Yang, Yi Sun, Junzhi Cui, Tianyu Guan, and Z. Yuan. A second-order two-scale algorithm for thermo-mechanical coupling problems in quasi-periodic porous materials. Communications in Computational Physics, 25(4), 2019. doi:10.4208/cicp.oa-2017-0252.

[52] Q. Yu and J. Fish. Temporal homogenization of viscoelastic and viscoplastic solids subjected to locally periodic loading. Computational Mechanics, 29(3):199-211, Sep 2002. doi:10.1007/ s00466-002-0334-y.

[53] Qing Yu and Jacob Fish. Multiscale asymptotic homogenization for multiphysics problems with multiple spatial and temporal scales: A coupled thermo-viscoelastic example problem. International Journal of Solids and Structures, 39(26):6429-6452, 2002. doi:10.1016/S0020-7683(02) 00255-X.

[54] H. W. Zhang, S. Zhang, J. Y. Bi, and B. A. Schrefler. Thermo-mechanical analysis of periodic multiphase materials by a multiscale asymptotic homogenization approach. International Journal for Numerical Methods in Engineering, 69(1):87-113, 2006. doi:10.1002/nme.1757. 